BULL. AUSTRAL. MATH. SOC.

VOL. 27 (1983), 319-320.

\title{
RAIKOV SYSTEMS AND ABSTRACT HARMONIC ANALYSIS
}

\author{
Jane Marie Lake
}

This thesis investigates the structure of Raikov systems and the implications of this structure for the algebraic properties of $M(G)$, the convolution algebra of finite Borel measures on the locally compact abelian group $G$.

The techniques used are those of modern abstract harmonic analysis and new examples of constructions are obtained of both unexpected pathology and desirable regular phenomena.

A compact perfect subset of $\Pi$ is a Dimichlet set if the constant function 1 can be uniformly approximated by continuous characters $\mathrm{X} \in \Pi^{\wedge} \backslash\{1\}$ on $A$. The second chapter will show that given any Dirichlet set $A$ on $\Pi$ there exists a singly generated Raikov system containing $A$ such that the Raikov system idempotent associated with this Raikov system is in the closure of the continuous characters $\overline{\Pi^{\wedge}} \subseteq \Delta M(\Pi)$.

The third chapter shows that for each $n \in \mathbb{Z}^{+}$the Raikov systems generated by $K_{n}$ subsets of $\mathbb{D}_{n}$ have associated Raikov system idempotent generalized characters lying in the closure of the continuous characters $\overline{\mathbf{D}_{n}^{n}}$ in the maximal ideal space $\Delta M\left(\mathbb{D}_{n}\right)$.

The fourth chapter answers a question of Graham and McGehee [3], p. 411, in the negative by constructing a continuous tame probability measure which is supported on a proper Raikov system on II. It also shows that there exists continuous tame probability measures in $M_{0}(G)$ concentrated on proper Raikov systems on $G$ where $G$ is a countable

Received 29 November 1982. Thesis submitted to University of New South Wales, March 1982. Degree approved October 1982. Supervisor: Professor Gavin Brown. 
product of cyclic groups.

The fifth chapter follows the work of Brown and Williamson [1] and define "churning" on $\mathbf{D}_{2}=\prod_{i=1}^{\infty}\left(\mathbb{Z}_{2}\right)_{i}$ and then studies some properties of measures under churning, and shows that $M_{0}\left(D_{2}\right)$ and $\operatorname{Rad} L^{1}\left(D_{2}\right)$ are not closed under churning.

\section{References}

[1] G. Brown and J.H. Williamson, "Rearranging measures", J. Austral. Math. Soc. Ser. A 34 (1983), 16-30.

[2] Charles F. Dunkl and Donald E. Ramirez, "Bounded projections on Fourier-Stieltjes transforms", Proc. Amer. Math. Soc. 31 (1972), $122-126$.

[3] Colin C. Graham, 0. Carruth McGehee, Essays in commutative harmonic analysis (Grundlehren der mathematischen Wissenschaften, 238. Springer-Verlag, Berlin, Heidelberg, New York, 1979).

Department of Mathematics,

University of New South Wales,

PO Box 1 ,

Kensington,

New South Wales 2033. 